\title{
The impact of using Pilates exercises on the electromyography of hyperlordosis for elderly
}

\section{Dr. Ehab Mohammed Emad El-Deen Ibrahim (*)}

The research aims to design a rehabilitation program using Pilates exercises for the hyperlodosis muscles for elderly. The researcher used semi-experimental method using the pre, inter and post measurement for one experimental group on a sample selected purposively, which consisted of (26) person with a percentage of $61.9 \%$ of the research community by (22) person who are the basic sample of the research and four persons who are the members of the survey sample of the research. The research results have shown that the positive impact of the rehabilitation program using Pilates exercises has led to an improvement for elderly with hyperlordosis in the variables under consideration ( length of the spine from different positions \& the electromyography of hyperlordosis to make muscular balance between the lumbar muscles specially "right, left " erector spinae muscle and "right, left" multifidus muscle with the abdominal muscles specially "right, left" external abdominal oblique muscle and "right, left" rectus abdominis muscle \& angles of hyperlordosis). The researcher recommends the need to apply the rehabilitation program using Pilates exercises under consideration on the elderly with hyperlordosis as well as a series of preventive measures to maintain the posture of the elderly.

Keywords: Kinetic Rehabilitation - Pilates - EMG - Lumbar spine 


\section{Introduction}

The elderly stage is the most age groups that need special care where we find that there are many physiological and morphological changes and many other changes that occur when the progress in life and affect clearly on the posture. At this stage, the amount of blood that is heading to the muscles decreases which causes not to have the full flow of nutrition, as well as lack of exercise that muscle makes a weakness and sometimes atrophy whenever advancing age. The muscle ends gradually cirrhosis and stick together and this is hampering some of the movements of the joints, which leads to take the wrong situations and thus postural deviations. (Kherbotaly, 2011; Saad, 2011)

When elderly have hyperlordosis, this is accompanied by weakness and prolong the abdominal muscles and the emergence of internal organs out of the sagging abdominal muscle, as the shortage and the strength in lumbar muscles and convergence of vertebral bodies from behind divergence from the front and occurs a shortening in ligaments of lumbar spine and become rigid, which in turn lead to numerous disorders in the vital organs in this area and reduces their ability to work. (Emad, 2016b)

It also found that Pilates exercises suit both genders with all ages and bodies, including elderly people without causing any health risk to them, they are also working to improve the strength and electrical activity of muscles and achieve efficiency in the flexible joints of the body in general and the flexibility of the spine in private and muscular balance between opposing muscle groups, which works to restore the body consistency. (Paterson, 2009)

It should be noted that there are some studies that recently studied the Pilates exercises in kinetic rehabilitation to restore muscle balance between opposite muscle groups, also the Pilates exercises used in rehabilitation of hyperlordosis of all segments of society, especially the elderly and with both genders, including the study of each of the (Irandoust \& Taheri, 2016; Yeon, 2016; Silva et al., 2015; Shahrjerdi , Golpayegani, Daghaghzadeh \& Karami, 2014; Rezaeei \& Ghofrani, 2012).

The results of a survey study conducted by the researcher indicated that $61.9 \%$ of elderly in the national project of pioneers' sports in Qalubia governorate are suffering from hyperlordosis and that led the researcher to the next question:

What is the impact of using Pilates exercises on the electromyography of hyperlordosis for elderly? 
The purpose of the study was to design a rehabilitation program using Pilates exercises for the muscles of the hyperlordosis for elderly under research by identifying:

1- The impact of the rehabilitation program for the muscles of the hyperlordosis on each of the (length of the spine in the different positions, electromyography of hyperlordosis \& angles of hyperlordosis).

2-Improvement ratios between the three research measurements (pre, inter \& post) in the (anthropometric spine, electromyography of hyperlordosis \& angles of hyperlordosis) for the experimental group under research.

We hypothesized the following:

1-There are statistically significant differences between the three research measurements (pre, inter \& post) in the favor of the post measurement in the variables of (length of the spine in different positions, electromyography of hyperlordosis \& angles of hyperlordosis) for the experimental group under research.

2-There are improvement ratios between the three research measurements (pre, inter\& post) in the variables of (anthropometric spine, electromyography of hyperlordosis \& angles of hyperlordosis) for the experimental group under research.

\section{Methods}

\section{Participants}

The researcher selected the research sample purposively which consisted of (26) persons with percentage $61.9 \%$ of the research community by (22) persons who are the basic sample members and four persons are members of the exploratory research sample of the same research community and outside the basic research sample.

Table (1) Statistical description of the research sample in age, weight and height variables $(\mathrm{N}=26)$

\begin{tabular}{c||c||c||c|c|c}
\hline \hline Variables & unit & Mean & Median & $\begin{array}{c}\text { Standard } \\
\text { Deviation }\end{array}$ & Skewness \\
\hline Age & Year & 60.3 & 60.5 & 3.4 & -0.18 \\
\hline Weight & $\mathrm{Kg}$ & 80.8 & 79.5 & 4.8 & 0.81 \\
\hline Height & $\mathrm{Cm}$ & 172.2 & 172 & 5.4 & 0.11 \\
\hline \hline
\end{tabular}

Table (1) indicates that the skewness coefficients for the variables of age, weight and height values confined between (-0.18: 0.81) which means that the skewness coefficients limited between \pm 3 which shows the moderation of the data.

Appendix (3) illustrates the moderation of the normal distribution of the data in the variables of (anthropometric spine, electromyography of hyperlordosis $\&$ angles of hyperlordosis) of the sample under research. 


\section{Measures}

1- Ristameter to measure the height in CM.

2- Medical scale to estimate the body weight in $\mathrm{kg}$.

3- E.M.G Wireless. Appendix (4)

4- Spinal mouse. Appendix (5)

\section{Procedures}

\section{A) Steps of designing of the Pilates exercises program}

The researcher reviewed the previous studies and scientific specialized references related to the research topic in order to design the Pilates exercises program for elderly with hyperlordosis and the following occurred:

\section{1- Identify aim of the rehabilitation program using Pilates exercises}

The rehabilitation program using Pilates exercises aims at the following:

A- Improve the length of the spine in different positions of the research sample.

B-Improve the measurements of the electromyography of hyperlordosis to make muscular balance between the lumbar and abdominal muscles of the research sample.

C-Improve the angles of hyperlordosis from upright position for the research sample.

\section{2- Identification of bases of the rehabilitation program using Pilates} exercises

A-To achieve the rehabilitation program its content target, which was designed for it.

B-To appropriate the rehabilitation program for the research sample while taking into consideration the scientific and physiological basis.

C-The possibility of implementing the rehabilitation program and its acceptance of the practical application.

D-To suit the rehabilitation program content with the total time and the number of specified units.

E-Taking into account that there is a continuity and sequence in the parts of the rehabilitation program.

F-Taking into account the order of the exercises of the rehabilitation programs in a way that helps the sequence of the muscle work between the muscle groups of the body parts in general and the deformity in particular.

G-Taking into account the basic principles of Pilates exercises such as (concentration, control, breathing, central, flow\& precision)

$\mathbf{H}$-Proper rationing of the variables of the rehabilitation load. 
I-Taking into account the security and safety factor in the selection of exercises.

3-Determine the general framework and the timetable of the distribution of the rehabilitation program using Pilates exercises

Table (2) General framework and the timetable of the distribution of the rehabilitation program using Pilates exercises

\begin{tabular}{|c|c|}
\hline Content & Timetable \\
\hline Duration of the program & Three months \\
\hline The number of weeks & 12 weeks \\
\hline Stages of the rehabilitation program & 3 stages \\
\hline $\begin{array}{l}\text { Number of weeks of each stage in the } \\
\text { rehabilitation program }\end{array}$ & $\begin{array}{l}\text { Four weeks for the first phase, four weeks for the } \\
\text { second phase \& four weeks for the third phase }\end{array}$ \\
\hline Time of one rehabilitation unit & Starts with $(55 \mathrm{~m})$ and ends with $(65 \mathrm{~m})$ \\
\hline The number of units of each program & 36 rehabilitation units \\
\hline $\begin{array}{l}\text { Time of the application of each stage } \\
\text { of the rehabilitation program }\end{array}$ & $\begin{array}{l}660 \mathrm{~m} \text { for the first phase, } 720 \mathrm{~m} \text { for the second } \\
\text { phase, } 780 \mathrm{~m} \text { for the third phase }\end{array}$ \\
\hline $\begin{array}{l}\text { Total time for the application of the } \\
\text { rehabilitation program }\end{array}$ & $2160 \mathrm{~m}$ (36 hours) \\
\hline $\begin{array}{l}\text { The implementation period of } \\
\text { rehabilitation units }\end{array}$ & Afternoon \\
\hline Arrange parts of rehabilitation unit & $\begin{array}{l}\text { A- Administrative works. } \\
\text { B- Watching videos and pictures about what will } \\
\text { be applied of Pilates exercises in a rehabilitation } \\
\text { unit. } \\
\text { C- Moving from the computer room to the Pilates } \\
\text { mat. } \\
\text { D- Warm-up. } \\
\text { E- The main part which contains the Pilates } \\
\text { exercises . } \\
\text { F- Cool -down. }\end{array}$ \\
\hline Appropriate load in program & Average \\
\hline
\end{tabular}

Table (2) illustrates the general framework and distribution of the timetable of the rehabilitation program using Pilates exercises for elderly, as the period of the rehabilitation program is 12 weeks divided into three stages, the number of the units in the rehabilitation program are (36) units a time of $(2160 \mathrm{~m})$ (36 hours) and the rehabilitative units applied afternoon using the average load. 


\section{4-Determine the content of the rehabilitation program using Pilates exercises}

The researcher reviewed the references and previous studies related to the designing of the rehabilitation programs and developed a set of Pilates exercises for hyperlordosis with identifying the aim of those exercises and divided them into progressive stages from easy to difficult. Appendix (1)

\section{B) Steps of the application of the rehabilitation program}

1-Pilot Study

The researcher conducted an exploratory study during the period from $10 / 7 / 2016$ to $14 / 7 / 2016$ on a sample consisting of four persons from the same research community and outside the basic sample in order to ensure the appropriateness of the rehabilitation program using Pilates exercises for the sample under research.

2-The Study of basic research

In the light of the outcome of the pilot study, the researcher applied the basic study of research in the following manner:

\section{A- Pre-measurements}

The researcher conducted the pre measurements of the "anthropometric spine, electromyography of hyperlordosis \& angles of hyperlordosis" on the sample under research in the period between $19 / 7 / 2016$ to $21 / 7 / 2016$.

B- The application of basic research study

The researcher applied the rehabilitation program using Pilates exercises on the sample under research in Benha club in the period from $24 / 7 / 2016$ to $23 / 10 / 2016$ by three rehabilitative units per week on (Sunday, Tuesday\& Thursday) for a period of three months. 


\section{Table (3) model of the rehabilitative unit using Pilates exercises to improve the electromyography of hyperlordosis for elderly}

\begin{tabular}{|c|c|c|c|c|c|c|c|}
\hline \multirow{2}{*}{$\begin{array}{c}\text { Aims of } \\
\text { Rehabilitation }\end{array}$} & \multirow{2}{*}{\multicolumn{5}{|c|}{$\begin{array}{l}\text { - Increase of oxygen flow to the working muscles } \\
\text { - Strengthening the abdominal muscles and lengthening the muscles } \\
\text { of the lumbar region }\end{array}$}} & Date & $1 / 9 / 2016$ \\
\hline & & & & & & Time & $60 \mathrm{~min}$ \\
\hline \multirow{3}{*}{\multicolumn{2}{|c|}{$\begin{array}{c}\text { Variables of } \\
\text { Load }\end{array}$}} & Intensity & \multicolumn{2}{|c|}{ Volume } & \multicolumn{3}{|c|}{ Density } \\
\hline & & \multirow{2}{*}{$(60 \%-65 \%)$} & Groups & Frequency & Between Groups & \multicolumn{2}{|c|}{ Between Frequency } \\
\hline & & & (3) & $(6-8)$ & $(60) \mathrm{s}$ & \multicolumn{2}{|c|}{$(15-30) s$} \\
\hline
\end{tabular}

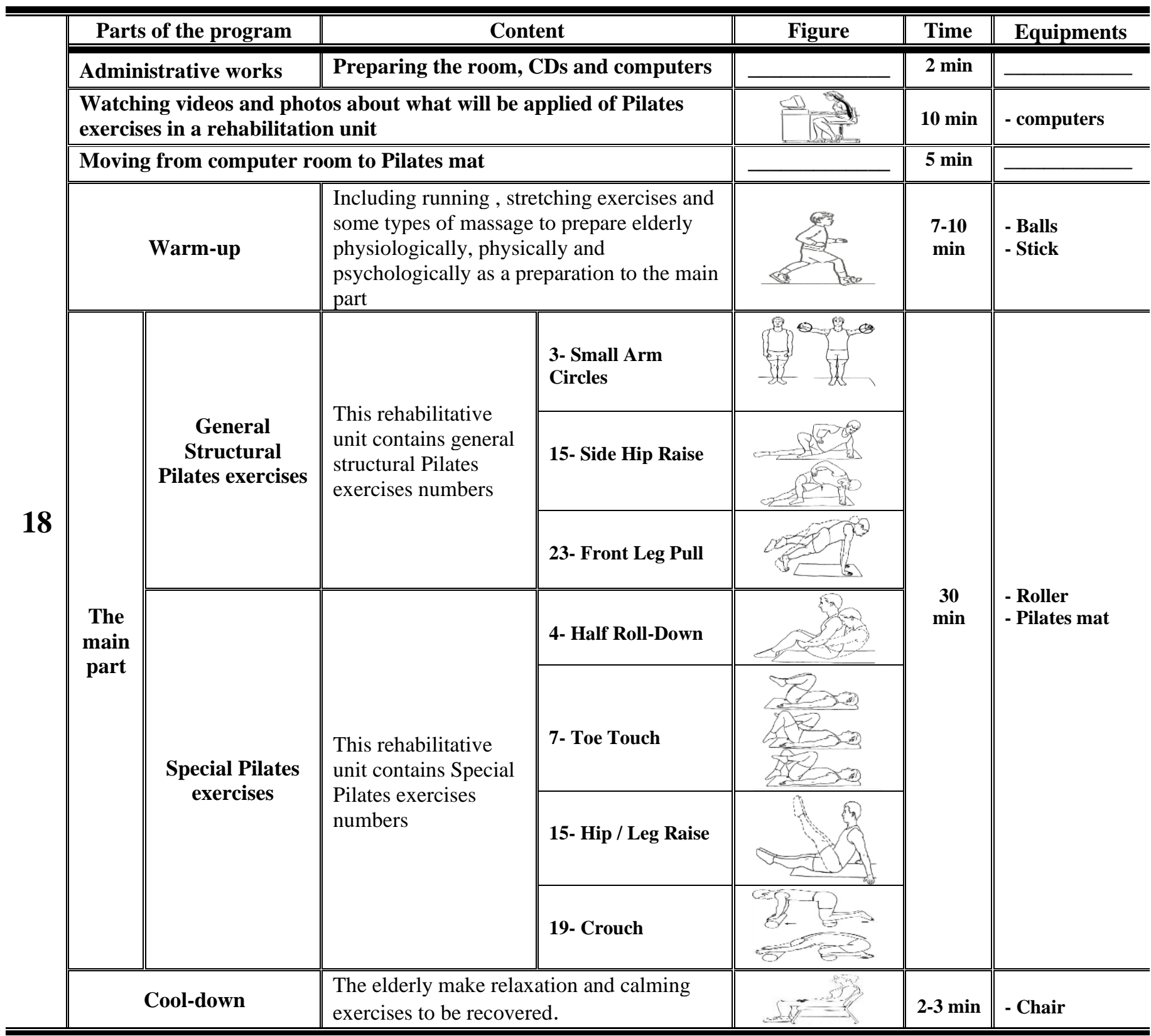


Appendix (2) illustrates the rehabilitation program using Pilates exercises to improve the electromyography of hyperlordosis for elderly.

C- Inter measurements

The researcher conducted the intermediate measurements of variables of "anthropometric spine, electromyography of hyperlordosis \& angles of hyperlordosis" on the sample under research in the period between 4/9/2016 to 6/9/2016.

D- Post measurements

The researcher conducted the post measurements of variables of "anthropometric spine, electromyography of hyperlordosis \& angles of hyperlordosis " on the sample under research in the period between $25 / 10 / 2016$ to $27 / 10 / 2016$.

\section{Statistical Analysis}

Data were processed by the following statistical methods:

- Arithmetic Mean

- Standard Deviation

- One-Way ANOVA

- The percentage of the absolute improvement \%
- Median

- Skewness

- L.S.D

\section{Results}

\section{- First hypothesis results}

Table (4) analysis of variance between three measurements of research in variables of "anthropometric spine, electromyography of hyperlordosis \& angles of hyperlordosis" for the experimental group.

\begin{tabular}{|c|c|c|c|c|c|c|c|}
\hline & Variables & Status & $\begin{array}{c}\text { Sum of } \\
\text { Squares }\end{array}$ & $\begin{array}{l}\text { Degrees of } \\
\text { freedom }\end{array}$ & $\begin{array}{c}\text { Mean } \\
\text { squares }\end{array}$ & $\mathbf{F}$ & Sig. \\
\hline \multirow{9}{*}{ 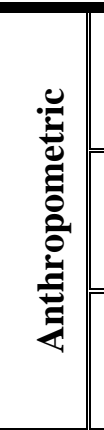 } & \multirow{3}{*}{$\begin{array}{l}\text { Spine Length } \\
\text { " Upright " }\end{array}$} & Between groups & 46.4 & 2 & 23.2 & \multirow{3}{*}{$9.8^{*}$} & \multirow{3}{*}{0.0} \\
\hline & & Within groups & 149 & 63 & 2.4 & & \\
\hline & & Total & 195.4 & 65 & & & \\
\hline & \multirow{3}{*}{$\begin{array}{l}\text { Spine Length } \\
\text { " Flexion " }\end{array}$} & Between groups & 87.8 & 2 & 43.9 & \multirow{3}{*}{$18.2^{*}$} & \multirow{3}{*}{0.0} \\
\hline & & Within groups & 151.7 & 63 & 2.4 & & \\
\hline & & Total & 239.5 & 65 & & & \\
\hline & \multirow{3}{*}{$\begin{array}{l}\text { Spine Length } \\
\text { " Extension " }\end{array}$} & Between groups & 55 & 2 & 27.5 & \multirow{3}{*}{$9.8^{*}$} & \multirow{3}{*}{0.0} \\
\hline & & Within groups & 176.8 & 63 & 2.8 & & \\
\hline & & Total & 231.8 & 65 & & & \\
\hline \multirow{9}{*}{ 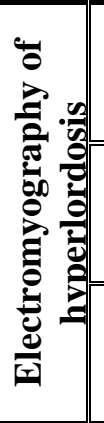 } & \multirow{3}{*}{ Right erector spinae } & Between groups & 1100 & 2 & 550 & \multirow{3}{*}{$58.3^{*}$} & \multirow{3}{*}{0.0} \\
\hline & & Within groups & 594 & 63 & 9.4 & & \\
\hline & & Total & 1694 & 65 & & & \\
\hline & \multirow{3}{*}{ Left erector spinae } & Between groups & 7999.6 & 2 & 399.8 & \multirow{3}{*}{$34.2^{*}$} & \multirow{3}{*}{0.0} \\
\hline & & Within groups & 736.2 & 63 & 11.7 & & \\
\hline & & Total & 1535.8 & 65 & & & \\
\hline & \multirow{3}{*}{ Right multifidus } & Between groups & 1090 & 2 & 545 & \multirow{3}{*}{$36^{*}$} & \multirow{3}{*}{0.0} \\
\hline & & Within groups & 953 & 63 & 15.1 & & \\
\hline & & Total & 2043 & 65 & & & \\
\hline
\end{tabular}




\begin{tabular}{|c|c|c|c|c|c|c|c|}
\hline & \multirow{3}{*}{ Left multifidus } & Between groups & 894.6 & 2 & 477.3 & \multirow{3}{*}{$38.9 *$} & \multirow{3}{*}{0.0} \\
\hline & & Within groups & 724 & 63 & 11.5 & & \\
\hline & & Total & 1618.6 & 65 & & & \\
\hline & \multirow{3}{*}{$\begin{array}{c}\text { Right external abdominal } \\
\text { oblique }\end{array}$} & Between groups & 616 & 2 & 308 & \multirow{3}{*}{$28.1 *$} & \multirow{3}{*}{0.0} \\
\hline & & Within groups & 690.5 & 63 & 11 & & \\
\hline & & Total & 1306.5 & 65 & & & \\
\hline & \multirow{3}{*}{$\begin{array}{l}\text { Left external abdominal } \\
\text { oblique }\end{array}$} & Between groups & 800 & 2 & 400 & \multirow{3}{*}{$54 *$} & \multirow{3}{*}{0.0} \\
\hline & & Within groups & 466.5 & 63 & 7.4 & & \\
\hline & & Total & 1266.5 & 65 & & & \\
\hline & \multirow{3}{*}{ Right rectus abdominis } & Between groups & 623.4 & 2 & 311.7 & \multirow{3}{*}{$50.9 *$} & \multirow{3}{*}{0.0} \\
\hline & & Within groups & 385.4 & 63 & 6.1 & & \\
\hline & & Total & 1008.8 & 65 & & & \\
\hline & \multirow{3}{*}{ Left rectus abdominis } & Between groups & 800 & 2 & 400 & \multirow{3}{*}{$49.8^{*}$} & \multirow{3}{*}{0.0} \\
\hline & & Within groups & 506.5 & 63 & 8 & & \\
\hline & & Total & 1306.5 & 65 & & & \\
\hline \multirow{21}{*}{ 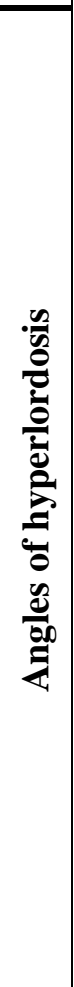 } & \multirow{3}{*}{$\begin{array}{l}\text { Thoracic Spine } 12 \text { / } \\
\text { Lumbar Spine } 1\end{array}$} & Between groups & 57.6 & 2 & 28.8 & \multirow{3}{*}{$31.1 *$} & \multirow{3}{*}{0.0} \\
\hline & & Within groups & 58.3 & 63 & 0.9 & & \\
\hline & & Total & 115.9 & 65 & & & \\
\hline & \multirow{3}{*}{ Lumbar Spine 1/2 } & Between groups & 57.4 & 2 & 28.7 & \multirow{3}{*}{$20.3 *$} & \multirow{3}{*}{0.0} \\
\hline & & Within groups & 89 & 63 & 1.4 & & \\
\hline & & Total & 146.4 & 65 & & & \\
\hline & \multirow{3}{*}{ Lumbar Spine 2/3 } & Between groups & 278.6 & 2 & 139.3 & \multirow{3}{*}{$49.9 *$} & \multirow{3}{*}{0.0} \\
\hline & & Within groups & 176 & 63 & 2.8 & & \\
\hline & & Total & 454.6 & 65 & & & \\
\hline & \multirow{3}{*}{ Lumbar Spine 3/4 } & Between groups & 176 & 2 & 88 & \multirow{3}{*}{$28.9 *$} & \\
\hline & & Within groups & 192 & 63 & 3 & & 0.0 \\
\hline & & Total & 368 & 65 & & & \\
\hline & & Between groups & 278.6 & 2 & 139.3 & & \\
\hline & Lumbar Spine 4/5 & Within groups & 176 & 63 & 2.8 & $49.9 *$ & 0.0 \\
\hline & & Total & 454.6 & 65 & & & \\
\hline & & Between groups & 278.6 & 2 & 139.3 & & \\
\hline & $\begin{array}{l}\text { Lumbar Spines/ } \\
\text { Sacrum 1 }\end{array}$ & Within groups & 204 & 63 & 3.2 & $43^{*}$ & 0.0 \\
\hline & & Total & 482.6 & 65 & & & \\
\hline & & Between groups & 6152.8 & 2 & 3076.4 & & \\
\hline & Total Lumbar Spine & Within groups & 1174.8 & 63 & 18.6 & $165^{*}$ & 0.0 \\
\hline & & Total & 7327.6 & 65 & & & \\
\hline
\end{tabular}

*Value of tabular "F" at significance level $(0.05)$ and degree of freedom $(2,63)=3.14$

Table (4) illustrates that are significant differences between the three research measurements (pre, inter \& post) in the variables under research. The calculated $(F)$ values were limited between (9.8: 18.2) in the variables of anthropometric spine, between (28.1: 58.3) in the variables of electromyography of hyperlordosis and between (20.3: 165) in the variables of angles of hyperlordosis. The calculated values were greater than the tabular value at the significance level (0.05). The calculated $\mathrm{P}$ - Values were less than their significance level. 
Table (5) test of less significant difference (L.S.D) between three measurements of research in variables of "anthropometric spine, electromyography of hyperlordosis \& angles of hyperlordosis" for the experimental group.

\begin{tabular}{|c|c|c|c|c|c|c|c|c|c|}
\hline & \multirow{2}{*}{ Variables } & \multirow{2}{*}{ Measurements } & \multirow[t]{2}{*}{ Mean } & \multicolumn{2}{|c|}{$\begin{array}{c}\text { Pre } \\
\text { measurement }\end{array}$} & \multicolumn{2}{|c|}{$\begin{array}{c}\text { Inter } \\
\text { measurement }\end{array}$} & \multicolumn{2}{|c|}{$\begin{array}{c}\text { Post } \\
\text { measurement }\end{array}$} \\
\hline & & & & $\begin{array}{c}\text { Mean } \\
\text { Difference } \\
\end{array}$ & Sig. & $\begin{array}{c}\text { Mean } \\
\text { Difference } \\
\end{array}$ & Sig. & $\begin{array}{c}\text { Mean } \\
\text { Difference } \\
\end{array}$ & Sig. \\
\hline \multirow{9}{*}{ 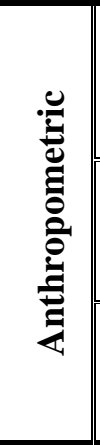 } & \multirow{3}{*}{$\begin{array}{l}\text { Spine Length } \\
\text { " Upright " }\end{array}$} & Pre measurement & 56 & & & $-1 *$ & 0.04 & $-2.1^{*}$ & 0.0 \\
\hline & & Inter measurement & 57 & & & & & $-1.1^{*}$ & 0.03 \\
\hline & & Post measurement & 58.1 & & & & & & \\
\hline & \multirow{3}{*}{$\begin{array}{l}\text { Spine Length } \\
\text { " Flexion " }\end{array}$} & Pre measurement & 60.2 & & & $-1.2 *$ & 0.02 & $-2.8^{*}$ & 0.0 \\
\hline & & Inter measurement & 61.4 & & & & & -1.6 & 0.0 \\
\hline & & Post measurement & 63 & & & & & & \\
\hline & \multirow{3}{*}{$\begin{array}{l}\text { Spine Length } \\
\text { "Extension " }\end{array}$} & Pre measurement & 54 & & & $-1^{*}$ & 0.04 & $-2.2^{*}$ & 0.0 \\
\hline & & Inter measurement & 55 & & & & & $-1.2^{*}$ & 0.02 \\
\hline & & Post measurement & 56.2 & & & & & & \\
\hline \multirow{24}{*}{ 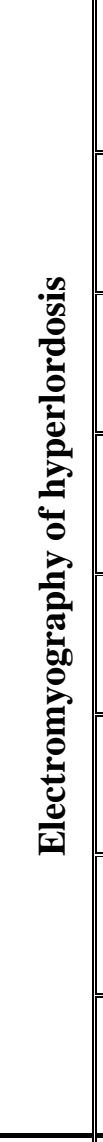 } & \multirow{3}{*}{$\begin{array}{l}\text { Right erector } \\
\text { spinae }\end{array}$} & Pre measurement & 36 & & & $5^{*}$ & 0.0 & $10^{*}$ & 0.0 \\
\hline & & Inter measurement & 31 & & & & & $5^{*}$ & 0.0 \\
\hline & & Post measurement & 26 & & & & & & \\
\hline & \multirow{3}{*}{ Left erector spinae } & Pre measurement & 34 & & & $4 *$ & 0.0 & $8.5^{*}$ & 0.0 \\
\hline & & Inter measurement & 30 & & & & & $4.5^{*}$ & 0.0 \\
\hline & & Post measurement & 25.5 & & & & & & \\
\hline & \multirow{3}{*}{ Right multifidus } & Pre measurement & 38 & & & $5 *$ & 0.0 & $10^{*}$ & 0.0 \\
\hline & & Inter measurement & 33 & & & & & $5 *$ & 0.0 \\
\hline & & Post measurement & 28 & & & & & & \\
\hline & \multirow{3}{*}{$\begin{array}{c}\text { Left } \\
\text { multifidus }\end{array}$} & Pre measurement & 36 & & & $5 *$ & 0.0 & 9* & 0.0 \\
\hline & & Inter measurement & 31 & & & & & $4 *$ & 0.0 \\
\hline & & Post measurement & 27 & & & & & & \\
\hline & \multirow{3}{*}{$\begin{array}{c}\text { Right external } \\
\text { abdominal oblique }\end{array}$} & Pre measurement & 18.5 & & & $-4 *$ & 0.0 & $-7.5^{*}$ & 0.0 \\
\hline & & Inter measurement & 22.5 & & & & & $-3.5^{*}$ & 0.0 \\
\hline & & Post measurement & 26 & & & & & & \\
\hline & \multirow{3}{*}{$\begin{array}{c}\text { Left external } \\
\text { abdominal oblique } \\
\end{array}$} & Pre measurement & 17 & & & $-4.5^{*}$ & 0.0 & $-8.5^{*}$ & 0.0 \\
\hline & & Inter measurement & 21.5 & & & & & $-4 *$ & 0.0 \\
\hline & & Post measurement & 25.5 & & & & & & \\
\hline & \multirow{3}{*}{$\begin{array}{c}\text { Right rectus } \\
\text { abdominis }\end{array}$} & Pre measurement & 17.5 & & & $-4^{*}$ & 0.0 & $-7.5^{*}$ & 0.0 \\
\hline & & Inter measurement & 21.5 & & & & & $-3.5^{*}$ & 0.0 \\
\hline & & Post measurement & 25 & & & & & & \\
\hline & \multirow{3}{*}{$\begin{array}{l}\text { Left rectus } \\
\text { abdominis }\end{array}$} & Pre measurement & 16 & & & $-4.5^{*}$ & 0.0 & $-8.5^{*}$ & 0.0 \\
\hline & & Inter measurement & 20.5 & & & & & $-4^{*}$ & 0.0 \\
\hline & & Post measurement & 24.5 & & & & & & \\
\hline \multirow{6}{*}{ 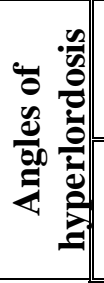 } & \multirow{3}{*}{$\begin{array}{l}\text { Thoracic Spine } 12 \\
\text { / Lumbar Spine } 1\end{array}$} & Pre measurement & -3.5 & & & $-1 *$ & 0.0 & $-2.3^{*}$ & 0.0 \\
\hline & & Inter measurement & -2.5 & & & & & $-1.3^{*}$ & 0.0 \\
\hline & & Post measurement & -1.2 & & & & & & \\
\hline & \multirow{3}{*}{ Lumbar Spine 1/2 } & Pre measurement & -3.3 & & & $-1 *$ & 0.0 & $-2.3^{*}$ & 0.0 \\
\hline & & Inter measurement & -2.3 & & & & & $-1.3^{*}$ & 0.0 \\
\hline & & Post measurement & -1 & & & & & & \\
\hline
\end{tabular}




\begin{tabular}{|c|c|c|c|c|c|c|}
\hline \multirow{3}{*}{ Lumbar Spine 2/3 } & Pre measurement & -11 & $-2 *$ & 0.0 & $-5^{*}$ & 0.0 \\
\hline & Inter measurement & -9 & & & $-3^{*}$ & 0.0 \\
\hline & Post measurement & -6 & & & & \\
\hline \multirow{3}{*}{ Lumbar Spine 3/4 } & Pre measurement & -11 & $-2 *$ & 0.0 & $-4 *$ & 0.0 \\
\hline & Inter measurement & -9 & & & $-2 *$ & 0.0 \\
\hline & Post measurement & -7 & & & & \\
\hline \multirow{3}{*}{ Lumbar Spine 4/5 } & Pre measurement & -13 & $-2 *$ & 0.0 & $-5^{*}$ & 0.0 \\
\hline & Inter measurement & -11 & & & $-3 *$ & 0.0 \\
\hline & Post measurement & -8 & & & & \\
\hline \multirow{3}{*}{$\begin{array}{l}\text { Lumbar Spine5/ } \\
\text { Sacrum } 1\end{array}$} & Pre measurement & -8 & $-2 *$ & 0.0 & $-5^{*}$ & 0.0 \\
\hline & Inter measurement & -6 & & & $-3 *$ & 0.0 \\
\hline & Post measurement & -3 & & & & \\
\hline \multirow{3}{*}{$\begin{array}{l}\text { Total Lumbar } \\
\text { Spine }\end{array}$} & Pre measurement & -49.8 & $-10^{*}$ & 0.0 & $-23.5^{*}$ & 0.0 \\
\hline & Inter measurement & -39.8 & & & $-13.5^{*}$ & 0.0 \\
\hline & Post measurement & -26.3 & & & & \\
\hline
\end{tabular}

Table (5) illustrates that there are statistically significant differences between three measurements of research in favor of the post measurement in variables of "anthropometric spine, electromyography of hyperlordosis $\&$ angles of hyperlordosis" for the experimental group under research and the calculated p-values were less than its significant level.

\section{- Second hypothesis results}

Table (6) the improvement ratios between three measurements of research in variables of "anthropometric spine, electromyography of hyperlordosis $\&$ angles of hyperlordosis" for the experimental group.

\begin{tabular}{|c|c|c|c|c|c|c|}
\hline & \multirow[b]{2}{*}{ Variables } & \multirow[b]{2}{*}{ Measurements } & \multirow{2}{*}{ Mean } & \multicolumn{3}{|c|}{ improvement ratios \% } \\
\hline & & & & $\begin{array}{c}\text { Pre } \\
\text { measurement }\end{array}$ & $\begin{array}{c}\text { Inter } \\
\text { measurement }\end{array}$ & $\begin{array}{c}\text { Post } \\
\text { measurement }\end{array}$ \\
\hline \multirow{9}{*}{ 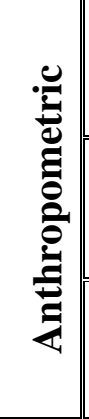 } & \multirow{3}{*}{$\begin{array}{l}\text { Spine Length } \\
\text { " Upright " }\end{array}$} & Pre measurement & 56 & & $1.8 \%$ & $3.8 \%$ \\
\hline & & Intra measurement & 57 & & & $1.9 \%$ \\
\hline & & Post measurement & 58.1 & & & \\
\hline & \multirow{3}{*}{$\begin{array}{l}\text { Spine Length } \\
\text { " Flexion " }\end{array}$} & Pre measurement & 60.2 & & $2 \%$ & $4.7 \%$ \\
\hline & & Intra measurement & 61.4 & & & $2.6 \%$ \\
\hline & & Post measurement & 63 & & & \\
\hline & \multirow{3}{*}{$\begin{array}{l}\text { Spine Length } \\
\text { "Extension " }\end{array}$} & Pre measurement & 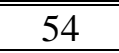 & & $1.9 \%$ & $4.1 \%$ \\
\hline & & Intra measurement & 55 & & & $2.2 \%$ \\
\hline & & Post measurement & 56.2 & & & \\
\hline \multirow{12}{*}{ 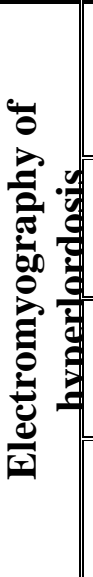 } & \multirow{3}{*}{ Right erector spinae } & Pre measurement & 36 & & $13.9 \%$ & $27.8 \%$ \\
\hline & & Intra measurement & 31 & & & $16.1 \%$ \\
\hline & & Post measurement & 26 & & & \\
\hline & \multirow{3}{*}{ Left erector spinae } & Pre measurement & 34 & & $11.8 \%$ & $25 \%$ \\
\hline & & Intra measurement & 30 & & & $15 \%$ \\
\hline & & Post measurement & 25.5 & & & \\
\hline & \multirow{3}{*}{ Right multifidus } & Pre measurement & 38 & & $13.2 \%$ & $26.3 \%$ \\
\hline & & Intra measurement & 33 & & & $15.2 \%$ \\
\hline & & Post measurement & 28 & & & \\
\hline & \multirow{3}{*}{ Left multifidus } & Pre measurement & 36 & & $13.9 \%$ & $25 \%$ \\
\hline & & Intra measurement & 31 & & & $12.9 \%$ \\
\hline & & Post measurement & 27 & & & \\
\hline
\end{tabular}




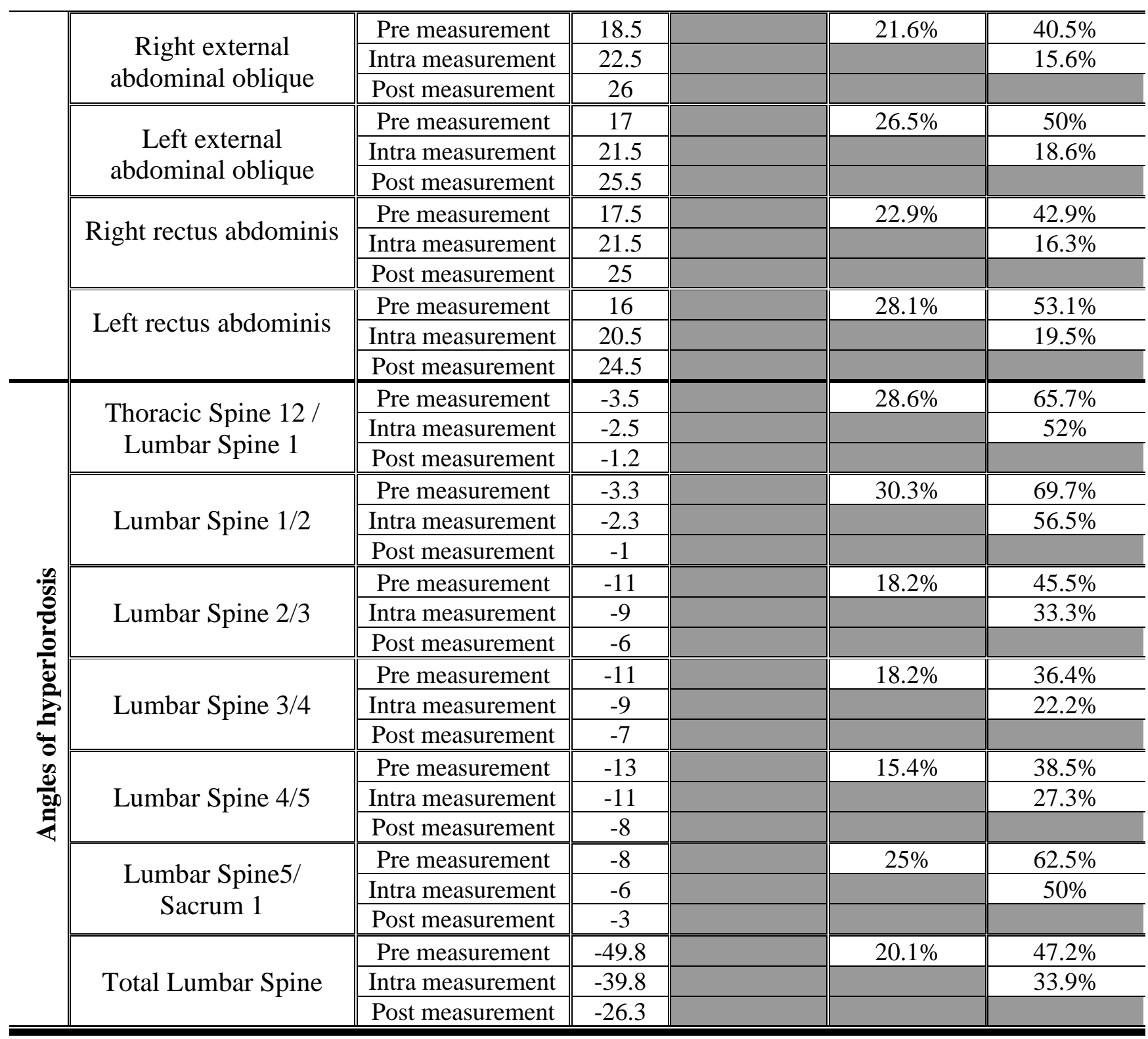

Table (6) illustrates that there are improvement ratios between research measurements in the three variables under research, where the highest percentage of improvement between the two measurements pre and post in the variable of anthropometric spine in the spine length when performing "Flexion" with a percentage of $4.7 \%$, the highest ratio of improvement between the two pre and post measurements in the electromyography of hyperlordosis variables in the left rectus abdominis muscle with a percentage of $53.1 \%$, followed by left external abdominal oblique muscle with a percentage of $50 \%$, followed by right rectus abdominis muscle with a percentage of $42.9 \%$,then the right external abdominal oblique with a rate of $40.5 \%$ and the highest percentage of improvement between the two pre and post measurements in the angles of hyperlordosis between the first and the second lumbar vertebra (Lumbar Spine 1/2 ) with a percentage of 69.7\%. 


\section{Discussion}

\section{- First hypothesis discussion}

Table (4, 5) illustrates that there are statistically significant differences between three measurements (pre, inter\& post) of research in favor of the post measurement in variables of anthropometric spine. The researcher attributes this to the positive impact of the rehabilitation program including Pilates exercises that working on adjusting the lumbar spine until reach to the normal status by the following:

1- Improved the postural and physiological state of the lumbar spine.

2- Improved the range of motion of the lumbar spine.

These results agreed with the results of a study of (Irandoust \& Taheri, 2016; Yeon, 2016) that the use of rehabilitation program using Pilates exercises for elderly works to improve the form and function of the spine including the "vertebrae, cartilage discs, joints \& ligaments" in addition to improving the form and function of the muscles of the front surface and the back of the spine in general and lumbar region in particular.

Table (4, 5) illustrates that there are statistically significant differences between three measurements (pre, inter\& post) of research in favor of the post measurement in variables of electromyography of hyperlordosis. The researcher attributes this to the positive impact of the rehabilitation program including Pilates exercises which led to:

1-Increase the efficacy of the sensory receptors and nerve excitement to muscles of the front surface of lumbar area such as (right external abdominal oblique, left external abdominal oblique, right rectus abdominis \& left rectus abdominis) and decrease of the efficacy of the sensory receptors and nerve excitement to muscles of the back surface of the lumbar area such as (right erector spinae muscle, left erector spinae muscle, right multifidus muscle\& left multifidus muscle).

2-Occurrence of muscular balance between the front and back surface of the lumbar area.

This agreed with the results of the study of (Silva et al., 2015) which showed that the use of Pilates exercises for elderly with hyperlordosis works on the occurrence of muscular balance between the front and back surface of the lumbar area. 
Table $(4,5)$ illustrates that there are statistically significant differences between three measurements (pre, inter\& post) of research in favor of the post measurement in variables of angles of hyperlordosis. The researcher attributes this to:

1- Good planning of the content of the rehabilitation program for elderly with hyperlordosis.

2- Follow the scientific bases when using the rehabilitation program for elderly with hyperlordosis.

3- The positive impact of the rehabilitation program including Pilates exercises which is repeated in accordance with the principles of Pilates with the application of dynamic and continuity to the variables of rehabilitation load.

It should be noted that the use of Pilates exercises for elderly with hyperlordosis works on the following:

1-Increase blood and oxygen flow to the working muscles.

2-lengthen the muscles of the spine especially the muscles of lumbar region.

3-Strengthening the abdominal muscles.

4-Re nervous muscular balance between the electromyography of the front and back surface of the lumbar area.

5- Improve angles of hyperlordosis. (Paterson, 2009)

\section{- Second hypothesis discussion}

Table (6) illustrates that there are improvement ratios between research measurements in the anthropometric variables of the spine, where the highest percentage of improvement between the two measurements pre and post measurements in the length of the spine when performing "Flexion" with a percentage of $4.7 \%$. The researcher attributes this to the positive impact of the rehabilitation program including special Pilates exercises, which are applied in the forward direction which led to the following:

1- Improved the biology of the lumbar region of the spine.

2- Improved the form and function of the muscles of the front surface and the back of the lumbar region, where the aim of Pilates exercises program is to lengthen the muscles of the back surface especially the muscles of the lumbar region and strengthen the muscles of the front surface especially the abdominal muscles.

Table (6) illustrates that there are improvement ratios between research measurements in the electromyography of hyperlordosis, where the highest percentage of improvement between the two measurements pre and post measurements in the electromyography of the left rectus abdominis muscle with a percentage of $53.1 \%$, then the left external abdominal oblique muscle at a rate of $50 \%$,then the electromyography 
of the right rectus abdominis with a rate of $42.9 \%$, then the electromyography of the right external abdominal oblique muscle with a rate of $40.5 \%$. The researcher attributes this to the positive impact of the rehabilitation program including Pilates exercises which led to:

1- Increase the electromyography of the muscles of the front surface of the lumbar area such as (right external abdominal oblique, left external abdominal oblique, right rectus abdominis \& left rectus abdominis) and decrease the electromyography of the muscles of the back surface of the lumbar area such as (right erector spinae muscle, left erector spinae muscle, right multifidus muscle \& left multifidus muscle).

2- Occurrence a balance between the electromyography of the muscles of the front surface with the electromygraphy of the muscles of the back surface of the lumbar area.

Table (6) illustrates that there are improvement ratios between research measurements in the angles of hyperlordosis, where the highest percentage of improvement between the two measurements pre and post measurements in the angle between the first and the second lumbar vertebrae (Lumbar Spine 1/2) with a percentage of 69.7\%. The researcher attributes this to the positive impact of the rehabilitation program as contained Pilates exercises that work on the improvement of the angles of the hyperlordosis, it became clear through the following:

1- The use of Pilates exercises numbers $(1,2,3,4,5,19,20 \& 27)$ led to an improvement in the angles of hyperlordosis specially the angle between (Thoracic Spine 12 / Lumbar Spine 1, Lumbar Spine $1 / 2$ \& Lumbar Spine 2/3)

2- The use of Pilates exercises numbers $(6,7,8,9,10,11,13,15,17$, $18,23,26,28 \& 29)$ led to an improvement in the angles of hyperlordosis specially the angle between (Lumbar Spine 3/4, Lumbar Spine 4/5 \& Lumbar Spine5/ Sacrum 1)

3- The use of Pilates exercises numbers $(12,14,16,21,22,24,25 \&$ 30) led to an improvement in the total angles of hyperlordosis.

These results agreed with the results of a study of both (Rezaeei \& Ghofrani, 2012; Shahrjerdi et al., 2014) that the use of Pilates exercises for elderly with hyperlordosis with codified scientific foundations work to improve the angles of hyperlordosis. 


\section{Conclusions and Applications}

In light of the aim of research and its hypotheses and within the nature of the sample and depending on the statistical analysis of the results and their explanation, the researcher found that the positive impact of the rehabilitation program using Pilates exercises led to an improvement in the variables of (length of the spine in the different positions, electromyography of hyperlordosis \& angles of hyperlordosis) for the experimental group under research and represent it in the following:

1- Increase the length of the spine from different positions (upright, flexion \& extension).

2- The balance of the muscles of the front surface with the muscles of the back surface of the lumbar area especially the balance of "right, left" external abdominal oblique muscle and the "right, left" rectus abdominis with the "right, left" erector spinae muscle and "right, left" multifidus muscle.

3- Improvement of the angles of hyperlordosis.

\section{Recommendations for future researches}

1-The need to apply the rehabilitation program using Pilates exercises under research on the elderly with hyperlordosis.

2-Establish a series of preventive measures to maintain the posture of the elderly.

3-The need for cooperation between the faculties of physical education and the ministry of state for sports affairs in the interest of the postural and physiological status for elderly.

4-Dissemination the health, nutritional and postural awareness for elderly.

5- Develop a national strategy aimed to ideal posture for elderly.

\section{References}

Emad, E.M. (2016). Modern Laboratory Measurements, Alexandria: The world of sport publishing foundation and Dar Al-wafaa for printing services, 225-234, 319-327.

Emad, E.M. (2016). Rehabilitation program for postural deformities, Alexandria: The world of sport publishing foundation and Dar Al-wafaa for printing services, 69.

Irandoust, K., Taheri, T. (2016). The impact of yoga and Pilates exercises on older adults, Iranian Journal of Ageing, Feb; 11(4):152-161.

Kherbotaly, S.S. (2011). Postural fitness and massage, Alexandria: Dar Al-Gameen for printing and binding, 32 . 
Paterson, J. (2009). Teaching Pilates for postural faults, illness \& injury, Butterworth Heinemann Elsevier, China, 45, 243-244.

Rezaeei, V., Ghofrani, M. (2012). Effect of two month Pilate's exercises on the lumbar hyperlordosis of 15-18 years old girl students, Annals of Biological Research, July; 3 (6):2667-2672.

Saad, S.B. (2011). Posture and ways to preserve it, Alexandria: Dar Alwafaa for printing and publishing, 62-63.

Shahrjerdi, S., Golpayegani, M., Daghaghzadeh, A., Karami.A. (2014). The effect of pilates-based exercises on pain, functioning and lumbar lordosis in women with non-specific chronic low back pain and hyperlordosis, The Scientific Journal of Zanjan University of Medical Science Nov; 22(94):120-131.

Silva, G., Morgan, M., Carvalho, W., Silva, E., Freitas, W., Silva, F., Souza,R.(2015).Electromyographic activity of rectus abdominis muscles during dynamic Pilates abdominal exercises, Journal of Bodywork \& Movement Therapies, Nov; 19(4):629-635.

Yeon, S. (2016). The effect of 12-week Pilates exercises on wellness in the elderly, Journal of Exercise Rehabilitation, April; 12(2):119-123. 